\title{
Development of a Novel Micro Biosensor for Monitoring Glutamate Release in the Brain
}

Abbreviated Title: Development of a Novel Glutamate Micro Biosensor

Yash M. Maniar ${ }^{1,2}$, Mallikarjunarao Ganesana ${ }^{2}, \mathrm{PhD}$, Elefterios Trikantzopolous ${ }^{2}$, and B. Jill

$$
\text { Venton }^{1,2}, \mathrm{PhD}
$$

\begin{abstract}
Affiliations:
${ }^{1}$ Neuroscience Distinguished Majors Program, University of Virginia, Charlottesville, VA 22903

${ }^{2}$ Department of Chemistry, University of Virginia, Charlottesville, VA 22903
\end{abstract}

Number of Pages: 25.

Number of Figures: 8.

Number of Words: Abstract, 247; Introduction, 621; Discussion, 520.

Conflict of Interest: The authors declare no competing financial interests.

Acknowledgements: The authors would like to thank the Center for Undergraduate Excellence for the Harrison Undergraduate Research Award and the NIH through grant R01NS076875 for their support. 


\section{ABSTRACT}

L-glutamate is a prevalent excitatory neurotransmitter in the central nervous system with important roles in a variety of normal neuronal function and also neurological and neurodegenerative diseases (Meldrum, 2000; Hamdan and Zain, 2014; Salazar et al., 2016). While many methods have been employed to detect glutamate levels in the brain, amperometric measurement with a glutamate oxidase-based biosensor offers numerous advantages including high spatial resolution and real-time measurement (Lee et al., 2007; Batra et al., 2016). However, commercially available glutamate biosensor models are expensive and unnecessarily large (Pinnacle Technology, Inc.). In this study, we characterized the performance of a novel, amperometric, glutamate oxidase-based micro biosensor with a $50 \mu \mathrm{m}$ platinum electrode and an o-phenylenediamine (o-PD) coating. An in vitro calibration curve was created, the linear range was found to reach $150 \mu \mathrm{M}$, and the sensitivity to glutamate within this range was 0.0966 $\mathrm{nA} / \mu \mathrm{M}$. The limit of detection was $0.044 \mu \mathrm{M}$. Selectivity testing with serotonin, adenosine, dopamine, glucose, uric acid, and ascorbic acid showed no biosensor response to these compounds. Shelf life stability was established but a 55\% decrease in sensitivity was measured over a 7-day period. Stimulated glutamate was measured successfully in the subthalamic nucleus of rat brain slices and in vivo. Higher stimulation pulse rates elicited increased glutamate release. In vitro post-calibration of the biosensor after use in brain tissue showed a $47 \%$ decrease in sensitivity to glutamate. This miniaturized glutamate biosensor shows promise for further studies in vivo and in disease states such as cerebral ischemia. 


\section{SIGNIFICANCE STATEMENT}

Glutamate is one of the most prevalent excitatory signaling molecules in the central nervous system and it plays a crucial role in a variety of neurological disorders including stroke and epilepsy (Meldrum 2000). The ability to monitor glutamate release real-time electrochemically in the brain would offer direct insight into the neurotransmitter's roles in these conditions. Current glutamate biosensor designs are prohibitively large for measurements in certain model organisms and expensive (Pinnacle Technology, Inc.). This study characterizes our amperometric microelectrode-based glutamate oxidase biosensor in vitro, in brain slices, and in vivo, establishing a better alternative that is cheaper and minimizes tissue damage. Our biosensor can now be used to study glutamate neurochemistry in disease states.

\section{INTRODUCTION}

Glutamate is one of the most prevalent excitatory signaling molecules in the central nervous system (CNS) (Salazar et al., 2016) and it plays a crucial role in a wide variety of brain functions such as memory, learning, and neurotransmission (Meldrum, 2000; Hamdan and Zain, 2014). At elevated concentrations, glutamate exhibits excitotoxic properties that are implicated in a variety of neurological disorders including ischemic stroke, epilepsy, and several neurodegenerative diseases (Mehta et al., 2013). Excitotoxicity is often mediated by glutamatergic overstimulation of N-methyl-D-aspartate (NMDA) receptors, resulting in excess calcium ion influx that can lead to neuronal death (Camacho and Massieu, 2006). The motivation 
to study glutamate in the brain arises from how much is yet unknown about its real-time characteristics in normal neuronal functioning and in disease states.

Multiple analytical techniques have been employed in the measurement of extracellular glutamate in the CNS, including microdialysis (Windels et al., 2003), chromatographic techniques such as HPLC (Clarke et al., 2007), and capillary electrophoresis (Vyas et al., 2011). However, these methods offer poor temporal resolution in comparison to electrochemical measurement using a glutamate biosensor (Lee et al., 2007). They additionally can require expensive equipment and extensive sample preparation, which can be time-consuming (Batra et al., 2016). The ability to monitor glutamate release electrochemically in real-time in the brain would offer direct insight into the role of the neurotransmitter in normal functioning and disease settings. However, glutamate is not electroactive and therefore undetectable with traditional electrochemical techniques.

Many glutamate biosensors utilize glutamate oxidase to enable real-time glutamate measurements in the brain amperometrically. Glutamate oxidase metabolizes glutamate and releases hydrogen peroxide as a by-product, which is electroactive and can be electrochemically detected (Özel et al., 2014). Glutamate dehydrogenase enzyme has also been employed in biosensor applications for the oxidation of glutamate; however, it has yielded longer response times and requires the co-immobilization of the $\mathrm{NAD}^{+}$cofactor (Hughes et al., 2016). A number of different glutamate biosensors have been developed in recent years for glutamate detection in different settings. A pencil graphite electrode with a zinc oxide nanorod composite showed a broad linear range for glutamate and long-term stability (Batra et al., 2016). However, this electrode is prohibitively large for in vivo applications. Another study deposited a chitosancarbon nanotube-gold nanowire coating on a glassy carbon electrode for glutamate determination 
in large food samples (Kitikul et al., 2017), but this biosensor is better suited for analyzing large samples through methods such as flow injection analysis. For physiological applications, platinum wire electrodes are common but most designs are quite large (O’Neill et al., 2008; Özel et al., 2014).

Current commercially available glutamate oxidase-based biosensor designs are also large (176 $\mu \mathrm{m}$ in diameter) for measurements in small model organisms and extremely expensive, starting at $\$ 210$ per sensor (Pinnacle Technology, Inc.). In this study, we report on the design and development of an amperometric, glutamate oxidase-based platinum microelectrode biosensor for real-time monitoring of glutamate in the mammalian brain. A smaller design would not only minimize tissue damage during biosensor implantation and removal, but it also would also allow more precise targeting of brain regions.

In this study, we developed a miniaturized glutamate microelectrode biosensor using a $50 \mu \mathrm{m}$ Pt wire. Prior to use, an o-PD polymer composite was electrocoated onto the Pt wire to act as a permselective membrane to selectively block common interfering substances in physiological conditions (Killoran and O’Neill, 2008; O’Neill et al., 2008). Biosensor analytical performance was tested amperometrically in a standard electrochemical cell and showed good sensitivity and selectivity. Our biosensor also showed excellent response time and limit of detection. The goals of this study were to employ the biosensor in rat brain slices and in in vivo applications, where we successfully measured stimulated glutamate in the rat subthalamic nucleus. 


\section{MATERIALS AND METHODS}

Biosensor Materials and in vitro reagents. Platinum wire $(50 \mu \mathrm{m}$ diameter, cat \#776000) and glass capillary tubes (4” long, $1.2 \mathrm{~mm} \times 0.68 \mathrm{~mm}$, cat \#627000) were obtained from A-M Systems. Polyimide capillaries (cat \#1068150022) with inner diameter $100.0 \mu \mathrm{m}$ were obtained from Polymicro Technologies. Silver conducting epoxy (cat \#8331) was obtained from MG Chemicals and 5-minute epoxy was obtained from Devcon. Model PE-21 glass microelectrode puller was obtained from Narishige. Glutamate oxidase $(0.1 \mathrm{U} / \mu \mathrm{L}$, cat \#80049) was obtained from Cosmo Bio Co., LTD. Ascorbate oxidase (200U/mL, cat \#J65782) was obtained from Alfa Aesar. Silver wire (cat \#317710010), acetic acid (glacial, cat \#423225000), L-(+)-ascorbic acid (99\%, cat \#105021000), uric acid (99+\%, cat \#171290250), adenosine (99+\%, cat \#164040250), dopamine (3-hydroxytyramine hydrochloride, 99\%, cat \#122000100), and serotonin hydrochloride (98+\%, cat \#215021000) were obtained from Acros Organics. Chitosan from shrimp shells (practical grade, cat \#417963), o-phenylenediamine (flaked, 99.5\%, cat \#P23938), albumin from bovine serum (cat \#A4737), L-glutamic acid ( $\geq 99 \%$ HPLC, cat \#G1251), and D(+)-glucose anhydrous (cat \#D9434) were obtained from Sigma-Aldrich. Isothesia (isoflurane, USP, cat \#029405) was obtained from Henry Schein. Sulfuric acid (certified ACS Plus, cat \#A300S-212) was obtained from Fisher Scientific.

Instrumentation. Amperometry and Cyclic Voltammetry experiments were performed using a Reference 600 Potentiostat (Gamry Instruments) with a modified Pt biosensor as the working electrode, an $\mathrm{Ag} / \mathrm{AgCl}$ (Pine research) reference electrode, and a Pt wire as a counter electrode. 
Fabrication of Biosensor. Platinum wire working electrodes were fabricated through a multistep process. For each electrode, a 4-5 $\mathrm{cm}$ in length strip of PTFE-coated platinum wire was cut and the Teflon coating was carefully removed. The Pt microelectrode was inserted into a $1.5 \mathrm{~cm}$ length of polyimide capillary with the biosensing active side as a protruding tip $1.5 \mathrm{~mm}$ in length. Then, 5-minute nonconductive epoxy was applied to seal the Pt wire-polyimide capillary interface, and the epoxy was allowed to cure for $30 \mathrm{~min}$ at room temperature. A glass capillary tube was pulled into two capillaries with a glass microelectrode puller and then the sharp end was polished. The polyimide capillary was inserted into the polished end of the glass capillary, with $1 \mathrm{~cm}$ of polyimide capillary protruding out. The polyimide capillary-glass capillary interface was sealed using 5-minute nonconductive epoxy. A $4 \mathrm{~cm}$ piece of silver wire with adhesive silver conductive epoxy was inserted into the other end of the glass capillary to form an electrical connection between the silver wire and the extended Pt wire inside the glass tube. Finally, a 5-minute nonconductive epoxy bubble was placed on the exposed biosensing end of the Pt wire, such that $1 \mathrm{~mm}$ in length of the microelectrode was exposed for the further modification steps.

Next, the microelectrodes were cleaned in $0.5 \mathrm{M}$ sulfuric acid using cyclic voltammetry by scanning from $-0.3 \mathrm{~V}$ to $1.5 \mathrm{~V}$ at $100 \mathrm{mV} / \mathrm{s}$ for 20 cycles. After the electrochemical treatment, the microelectrode was rinsed thoroughly with distilled water. The clean electrode was electrocoated with o-PD by applying a potential of $+0.7 \mathrm{~V}$ in a stirred solution consisting of $300 \mathrm{mM} \mathrm{o-}$ PD in PBS buffer at $\mathrm{pH} 7.4$ for 10 minutes. This method produces a thin, perm-selective and self-sealing film on the electrode surface (Killoran and O'Neill, 2008). Then, a 1:2 mixture of glutamate oxidase: $1 \mathrm{wt} \%$ chitosan in $0.1 \mathrm{M}$ acetic acid was prepared. Two $1.5 \mu \mathrm{L}$ aliquots of this mixture were manually deposited on the working area of the Pt wire and allowed to dry. A 
total of $2 \mu \mathrm{L}$ of $200 \mathrm{U} / \mathrm{mL}$ ascorbate oxidase was manually deposited on to the $\mathrm{Pt}$ wire and allowed to dry. The biosensors were then stored in a refrigerator until use. In the final step prior to use, the electrode was dipped in $5 \mathrm{mg} / \mathrm{mL}$ bovine serum albumin for one minute to avoid any non-specific adsorption.

Electrochemical Measurements. Biosensor performance was characterized using amperometry at an applied potential of $+0.6 \mathrm{~V}$ in vitro, in brain slices, and in vivo. Glutamate was manually injected into the electrochemical cell (stirring solution of PBS buffer at $\mathrm{pH} 7.4$ ) in in vitro studies. Selectivity testing was conducted by injecting the following interfering species found in the brain: $100 \mathrm{nM}$ serotonin, $1 \mu \mathrm{M}$ adenosine, $1 \mu \mathrm{M}$ dopamine, $0.5 \mathrm{mM}$ glucose, $100 \mu \mathrm{M}$ uric acid, and $200 \mu \mathrm{M}$ ascorbic acid.

Brain Slice Experiments. All protocols for animal experiments were approved by the Animal Care and Use Committee at the University of Virginia. Male Sprague-Dawley rats (250-350g) were housed in a vivarium and given food and water ad libetum. Rats were anesthetized with isoflurane and beheaded. $400 \mu \mathrm{m}$ thick sagittal slices containing the target region, the subthalamic nucleus, were collected in oxygenated artificial cerebral spinal fluid (aCSF) (Pajski and Venton, 2010). Once collected, slices were heated in a water bath to $37^{\circ} \mathrm{C}$ to equilibrate for at least 30 minutes. During experimentation, oxygenated aCSF was perfused over the slice at 2 $\mathrm{mL} / \mathrm{min}$. A biphasic stimulating electrode rested on top of the tissue on the sub-thalamic nucleus (approximate coordinates $2.9 \mathrm{~mm}$ mediolateral, $7.6 \mathrm{~mm}$ dorsoventral, $-3.5 \mathrm{~mm}$ anteroposterior). The working electrode was implanted between the prongs of the stimulating electrode and about $500 \mu \mathrm{m}$ away. It was implanted through the tissue to maximize the surface area of the electrode 
that is in direct contact with the tissue. The stimulation parameters were 10-50 one (1) ms biphasic pulses of $500 \mu \mathrm{A}$ at a frequency of $120 \mathrm{~Hz}$ (Windels et al., 2000).

In Vivo Experiments and Surgery. Male Sprague-Dawley Rats (250-350 g; Charles-River, Wilmington, MA, USA) were anesthetized with 50\% wt urethane (Sigma Aldrich, $\geq 99 \%$, cat \#U2500) solution in saline (Baxter; Deerfield, IL, USA) (1.5 g/kg, i.p). The surgical site was shaved and $0.25 \mathrm{~mL}$ of bupivicaine (Sensorcaine MPF, APP Pharmaceuticals, LLC;

Schaumburg, IL, USA) was administered subcutaneously for local analgesia. After exposing the skull, holes were drilled for placement of electrodes using a stereotaxic drill. Stimulated glutamate measurements were conducted in subthalamic nucleus using the coordinates (in mm from bregma): anterior-posterior (AP): -3.5 , mediolateral (ML): +2.4 , and dorsoventral (DV):

-7.5 . Both working and stimulating electrodes were placed in the same region with $100 \mu \mathrm{m}$ apart. $\mathrm{The} \mathrm{Ag} / \mathrm{AgCl}$ reference electrode was inserted on the contralateral side of the brain. The rat's body temperature was maintained at $37^{\circ} \mathrm{C}$ using a heating pad with a thermistor probe ( $\mathrm{FHC}$, Bowdoin, ME, USA). Stimulated glutamate release was measured by applying stimulation pulse trains $(500 \mu \mathrm{A}, 30$ or 50 pulses, $120 \mathrm{~Hz})$, and the glutamate response was recorded in the subthalamic nucleus.

\section{RESULTS}

\section{Biosensor Preparation and Operation}

An operational schematic of the glutamate biosensor is shown in Figure 1. After electrochemical cleaning of the Pt wire, a thin, perm-selective o-PD membrane was 
electrochemically deposited on the wire using amperometry. o-PD has been shown to block interferences from most large interfering compounds (Killoran and O'Neill, 2008). Glutamate oxidase immobilized in a biocompatible chitosan mixture was then deposited manually onto the electrode. This enzyme catalyzes the oxidation of glutamate, a reaction that releases electrochemically active hydrogen peroxide. Peroxide undergoes a redox reaction at the $\mathrm{Pt}$ surface, which causes a transfer of electrons that can be detected as current using amperometry. Because ascorbate is one of the primary interferents in the central nervous system, at concentrations in the range of $400 \mu \mathrm{M}$ (Miele and Fillenz, 1996), ascorbate oxidase was also manually deposited to eradicate any potential ascorbic acid interference. The biosensors were rinsed in bovine serum albumin prior to use to avoid non-specific adsorption of proteins during in vivo applications.

\section{Biosensor Calibration}

All measurements of glutamate biosensor and its optimization was carried out in standard solutions of PBS by successive additions of glutamate and current response was measured using amperometry at a constant potential of $+0.6 \mathrm{~V}$ in standard electrochemical cell. Glutamate injections were done in triplicates, starting from $5 \mu \mathrm{M}$ and increasing in increments of $5 \mu \mathrm{M}$ up until $50 \mu \mathrm{M}$. A representative amperogram showing the response of glutamate to successive additions of glutamate was shown in Figure 2. A linear calibration curve was obtained in the range of 5-1500 $\mu \mathrm{M}$ (Figure 3A) and the biosensor showed a good linear relationship for glutamate concentrations in the range of 5-150 $\mu \mathrm{M}$ (Figure 3B) with a sensitivity of 0.0966 $\mathrm{nA} / \mu \mathrm{M}$. Limit of detection (LOD) of the present biosensor was $0.044 \mu \mathrm{M}(\mathrm{S} / \mathrm{N}=6)$, and was calculated using the formula $\mathrm{LOD}=3(\mathrm{SD} / \mathrm{m})$ where $\mathrm{SD}=$ standard deviation of the 
amperometric signal of PBS, and $\mathrm{m}=$ slope of the calibration curve obtained from Figure 3B, which is lower than that of a previous glutamate biosensor (Özel et al., 2014). The linear range of the biosensor is more than sufficient for in vivo applications in brain studies, since the glutamate concentration in the rat brain is only on the order of a few micromolar (Hamdan and Zain, 2014). The biosensor has short response time and a steady state limiting current value was reached in less than $2 \mathrm{~s}$ after the addition of glutamate, indicating a very good diffusion rate of the substrate through the chitosan matrix to the active immobilized materials and the electrode surface.

\section{Biosensor Selectivity and Shelf Life}

In the CNS, the biosensor will be exposed to a variety of interferences besides glutamate. Successful functionality of the biosensor in in vivo CNS glutamate measurements relies on the biosensor targeting glutamate selectively and detecting it alone. The biosensor design incorporates a perm-selective o-PD membrane, chitosan polymer matrix and ascorbate oxidase, which are all known to reject ascorbic acid as well as other interfering species. In order to ensure that the biosensor was responsive to only glutamate, but not to any other common electroactive analytes found in the CNS, selectivity testing was conducted in vitro using $100 \mathrm{nM}$ serotonin, $1 \mu \mathrm{M}$ adenosine, $1 \mu \mathrm{M}$ dopamine, $0.5 \mathrm{mM}$ glucose, $100 \mu \mathrm{M}$ uric acid, and $200 \mu \mathrm{M}$ ascorbic acid. The biosensor showed a negligible or no response to any of these analytes, indicating no interference was detected from these analytes (Figure 4). Current response to 20 $\mu \mathrm{M}$ glutamate was tested before and after testing the other analytes, and the biosensor did not show any change in current response to glutamate. This indicates that biosensor can reliably measure glutamate in the brain even after exposure to the other analytes. These results suggest 
that exposure to other CNS analytes does not affect the performance of the biosensor and its sensitivity to glutamate.

Another important characteristic of the biosensor is storage stability. Shelf life studies were conducted to measure the change in glutamate sensitivity over a 7-day period under dry conditions stored at $+4^{\circ} \mathrm{C}$ (Figure 5) Starting with Day 0 as the day of enzyme deposition, the biosensor's response to five $5 \mu \mathrm{M}$ glutamate injections was measured on each day from Day 1 to Day 7. New biosensors were used for the measurements on each day and biosensors from different batches were tested to show the variability between the batches. Figure 5 shows a moderate $(55 \%)$ loss in sensitivity over a 7 -day period.

\section{Application of the Biosensor in Rat Brain Slice and In Vivo}

To demonstrate the potential use of these biosensors for real-time biological applications, the functionality of the biosensor was further demonstrated in both rat brain slices and the in vivo rat brain for measuring stimulated glutamate release. Preliminary results show the demonstration of biosensor testing in the subthalamic nucleus of rat sagittal slices. Biphasic stimulations were carried out using Dagon BSI-960 biphasic stimulus isolator with different stimulation pulse rates. The stimulation pulse rates were varied between 10,30, and 50 pulses. Current response of the biosensor to stimulated glutamate release was measured using the amperometry as mentioned in the Methods. Greater stimulation pulse rates led to greater current response in the slice (Figure 6). The inset overlays the current response to the stimulated glutamate release peaks allowing for direct comparison of pulse rate responses.

Biosensor performance in the intact rain brain in vivo was tested in the subthalamic nucleus of the anaesthetized rat brain. Glutamate was stimulated with stimulation pulse rates of 
either 30 or 50 pulses. Again, greater stimulation pulse rates elicited a larger current response; two separate in vivo experiments were shown in Figure 7. The inset overlays the stimulation peaks allowing for direct comparison of pulse rate responses. The sharp current spike just prior to stimulation was the electrical noise generated from stimulator. Peak rise after the stimulation was the actual biosensor response to the release of glutamate. Preliminary in vivo measurements in both brain slices and intact rat brains demonstrates the potential use of these biosensors to investigate the mechanisms of glutamate neurotransmission for several neurological diseases, such as ischemia, Alzheimer's and Parkinson's diseases.

Biosensor sensitivity after brain slice and in vivo applications was tested after exposure to the brain environment. In vitro post-calibrations after the sensors had been used either in slices or in vivo were performed, and the response characteristics are compared to pre-calibrations in Figure 8. While the shape of the response amperogram was similar for both pre and post calibrations (Figure 8A), Figure $8 \mathrm{~B}$ biosensors showed a $47 \%$ difference in sensitivity to glutamate after use in the brain.

\section{DISCUSSION}

This present study demonstrates the ability to fabricate and characterized the miniaturized micro biosensor. With a small design featuring a $50 \mu \mathrm{m}$ in diameter Pt wire, our biosensor successfully showed strong analytical performance with sensitivity to glutamate of 0.0966 $\mathrm{nA} / \mu \mathrm{M}$ and a linear range from 5-150 $\mu \mathrm{M}$. The o-PD polymer and ascorbate oxidase coatings were successful at inhibiting a number of potential interfering analytes. Selectivity testing in vitro enables us to be confident about detecting glutamate selectively in physiological conditions, 
without concern for interference. Additionally, we were able to show that exposure to other compounds does not affect the biosensor's sensitivity to glutamate. Biosensor shelf life stability was established over a 7-day period, and the decrease in sensitivity that was observed over the course of a week was within acceptable limits. Application of the biosensor for biological applications resulted in successful measurements of glutamate levels both rat brain slices and in intact rats in the subthalamic nucleus region of the brain.

The commercially available glutamate biosensor reports a linear range only up to $50 \mu \mathrm{M}$ (Pinnacle Technology, Inc.), which is much smaller than that of our biosensor. Although $50 \mu \mathrm{M}$ is sufficient for normal physiological glutamate levels (Hamdan and Zain, 2014), a larger linear range can be useful for in vitro studies and overstimulation experiments. One study used the Pinnacle glutamate biosensor to measure glutamate during in vivo high frequency stimulation of the rat subthalamic nucleus (Lee et al., 2007). Although the Pinnacle biosensor showed linearity only up to $50 \mu \mathrm{M}$, these authors used it for stimulated glutamate release beyond the end of this range. Biosensors with larger linearity would have been highly useful for this study and our biosensor, with a linear range up to $150 \mu \mathrm{M}$, would have been more applicable. In the future, our biosensor can be adapted to similar studies. In addition, the authors noted that tissue damage caused by biosensor implantation was an experimental limitation. Our miniaturized biosensor would minimize tissue damage and would be a highly useful alternative to existing commercial biosensor options.

As an initial characterization of a novel miniaturized glutamate biosensor, this study has been able to evaluate biosensor sensitivity, selectivity, shelf life stability, and successfully measure glutamate in brain slices and in vivo. While these results are promising, we would like to establish more robust measurements for in vivo applications and increase the number of trials 
to provide more support for use of the biosensor in wider biomedical and clinical applications. Ultimately, the goal of this biosensor is for brain slice and in vivo use in disease studies. In these pursuits, our micro biosensor will prove useful in minimizing tissue damage and targeting small regions of the brain. Our primary goal is to continue brain experiments, explore glutamate in different brain regions, and eventually study glutamate excitotoxicity in ischemic stroke conditions. Finally, our lab is interested in adenosine as potential neuromodulator of glutamate during neurological conditions, such as cerebral ischemia (Boison, 2008). Our biosensor can be used to directly measure the impact of adenosine on brain glutamate levels in real-time. These research goals provide numerous potential applications of our glutamate biosensor.

\section{REFERENCES}

Batra B, Yadav M, Pundir CS (2016) 1-Glutamate biosensor based on 1-glutamate oxidase immobilized onto $\mathrm{ZnO}$ nanorods/polypyrrole modified pencil graphite electrode. Biochem Eng J 105:428-436.

Boison D (2008) Adenosine as a neuromodulator in neurological diseases. Curr Opin Pharmacol $8: 2-7$.

Camacho A, Massieu L (2006) Role of Glutamate Transporters in the Clearance and Release of Glutamate during Ischemia and its Relation to Neuronal Death. Arch Med Res 37:11-18.

Clarke G, O’Mahony S, Malone G, Dinan TG (2007) An isocratic high performance liquid chromatography method for the determination of GABA and glutamate in discrete regions of the rodent brain. J Neurosci Methods 160:223-230.

Hamdan SK, Zain ZM (2014) In vivo electrochemical biosensor for brain glutamate detection: A 
mini review. Malaysian J Med Sci 21:11-25.

Hughes G, Pemberton RM, Fielden PR, Hart JP (2016) The design, development and application of electrochemical glutamate biosensors. TrAC Trends Anal Chem 79:106-113.

Killoran SJ, O'Neill RD (2008) Characterization of permselective coatings electrosynthesized on Pt-Ir from the three phenylenediamine isomers for biosensor applications. Electrochim Acta 53:7303-7312.

Kitikul J, Satienperakul S, Preechaworapun A, Pookmanee P, Tangkuaram T (2017) A Simple Flow Amperometric Electrochemical Biosensor Based on Chitosan Scaffolds and Gold Nanowires Modified on a Glassy Carbon Electrode for Detection of Glutamate in Food Products. Electroanalysis 29:264-271.

Lee KH, Kristic K, van Hoff R, Hitti FL, Blaha C, Harris B, Roberts DW, Leiter JC (2007) High-frequency stimulation of the subthalamic nucleus increases glutamate in the subthalamic nucleus of rats as demonstrated by in vivo enzyme-linked glutamate sensor. Brain Res 1162:121-129.

Mehta A, Prabhakar M, Kumar P, Deshmukh R, Sharma PL (2013) Excitotoxicity: Bridge to various triggers in neurodegenerative disorders. Eur J Pharmacol 698:6-18.

Meldrum BS (2000) Glutamate as a neurotransmitter in the brain: review of physiology and pathology. J Nutr 130:1007S-15S.

Miele M, Fillenz M (1996) In vivo determination of extracellular brain ascorbate. J Neurosci Methods 70:15-19.

O’Neill RD, Rocchitta G, McMahon CP, Serra PA, Lowry JP (2008) Designing sensitive and selective polymer/enzyme composite biosensors for brain monitoring in vivo. TrAC Trends Anal Chem 27:78-88. 
Özel RE, Ispas C, Ganesana M, Leiter JC, Andreescu S (2014) Glutamate oxidase biosensor based on mixed ceria and titania nanoparticles for the detection of glutamate in hypoxic environments. Biosens Bioelectron 52:397-402.

Pajski ML, Venton BJ (2010) Adenosine release evoked by short electrical stimulations in striatal brain slices is primarily activity dependent. ACS Chem Neurosci 1:775-787.

Salazar P, Martín M, O’Neill RD, González-Mora JL (2016) Glutamate microbiosensors based on Prussian Blue modified carbon fiber electrodes for neuroscience applications: In-vitro characterization. Sensors Actuators B Chem 235:117-125.

Vyas CA, Rawls SM, Raffa RB, Shackman JG (2011) Glutamate and aspartate measurements in individual planaria by rapid capillary electrophoresis. J Pharmacol Toxicol Methods 63:119-122.

Windels F, Bruet N, Poupard A, Feuerstein C, Bertrand A, Savasta M (2003) Influence of the frequency parameter on extracellular glutamate and $\gamma$-aminobutyric acid in substantia nigra and globus pallidus during electrical stimulation of subthalamic nucleus in rats. J Neurosci Res 72:259-267.

Windels F, Bruet N, Poupard A, Urbain N, Chouvet G, Feuerstein C, Savasta M (2000) Effects of high frequency stimulation of subthalamic nucleus on extracellular glutamate and GABA in substantia nigra and globus pallidus in the normal rat. Eur J Neurosci 12:4141-4146. 


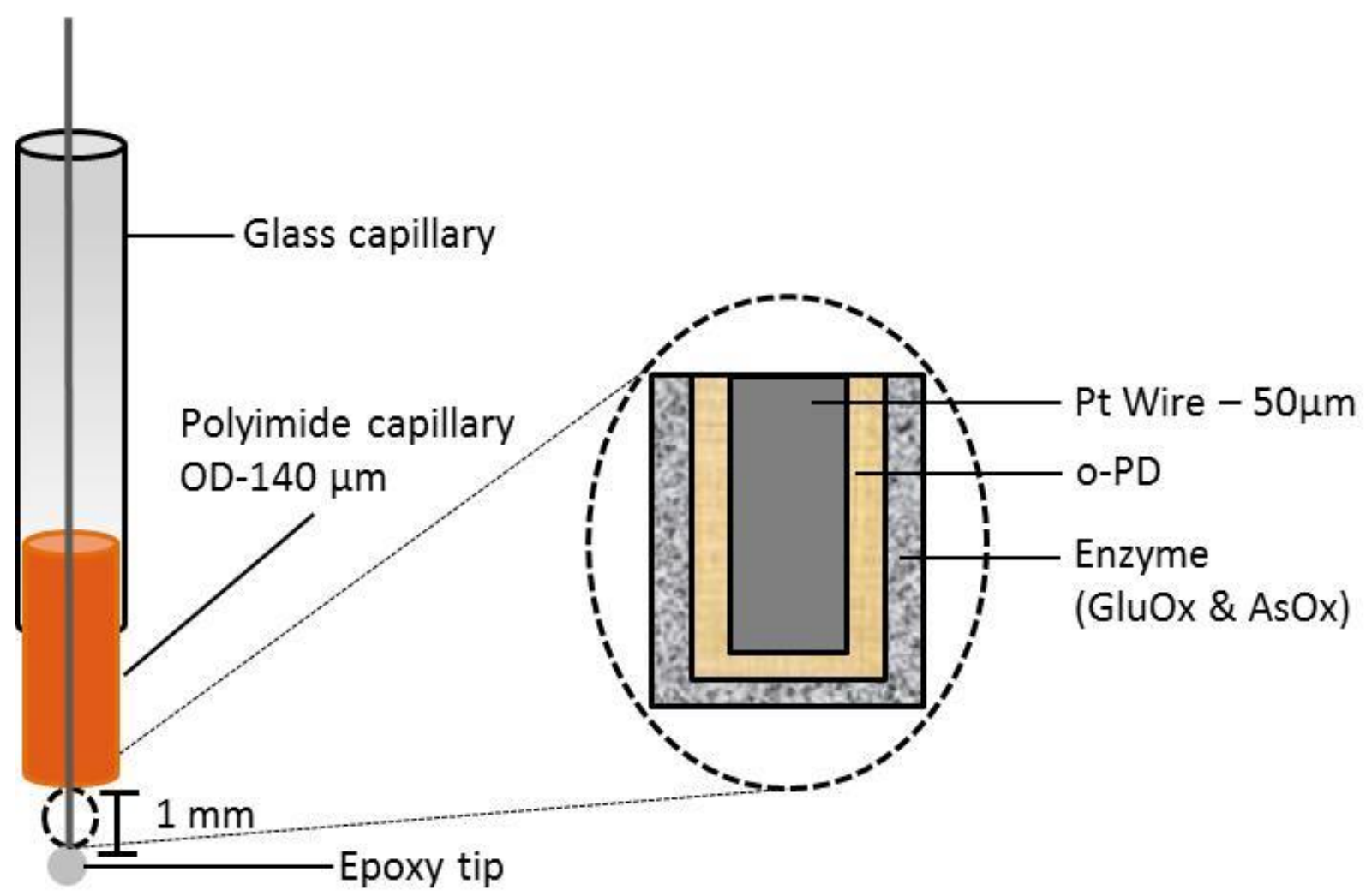

Figure 1. Operational schematic of Pt glutamate biosensor. The Pt wire has a $50 \mu \mathrm{m}$ diameter and is coated in an o-PD polymer composite, a Glutamate Oxidase/chitosan mixture (GluOx), and Ascorbate Oxidase (AsOx). The wire has an epoxy tip at the protruding end to aid in enzyme deposition. The Pt wire is sheathed in a polyimide capillary, which is subsequently sheathed in a glass capillary tube. The top end of the Pt wire is connected to a silver wire (not shown) via silver conducting epoxy; this silver wire makes contact with the Potentiostat. 


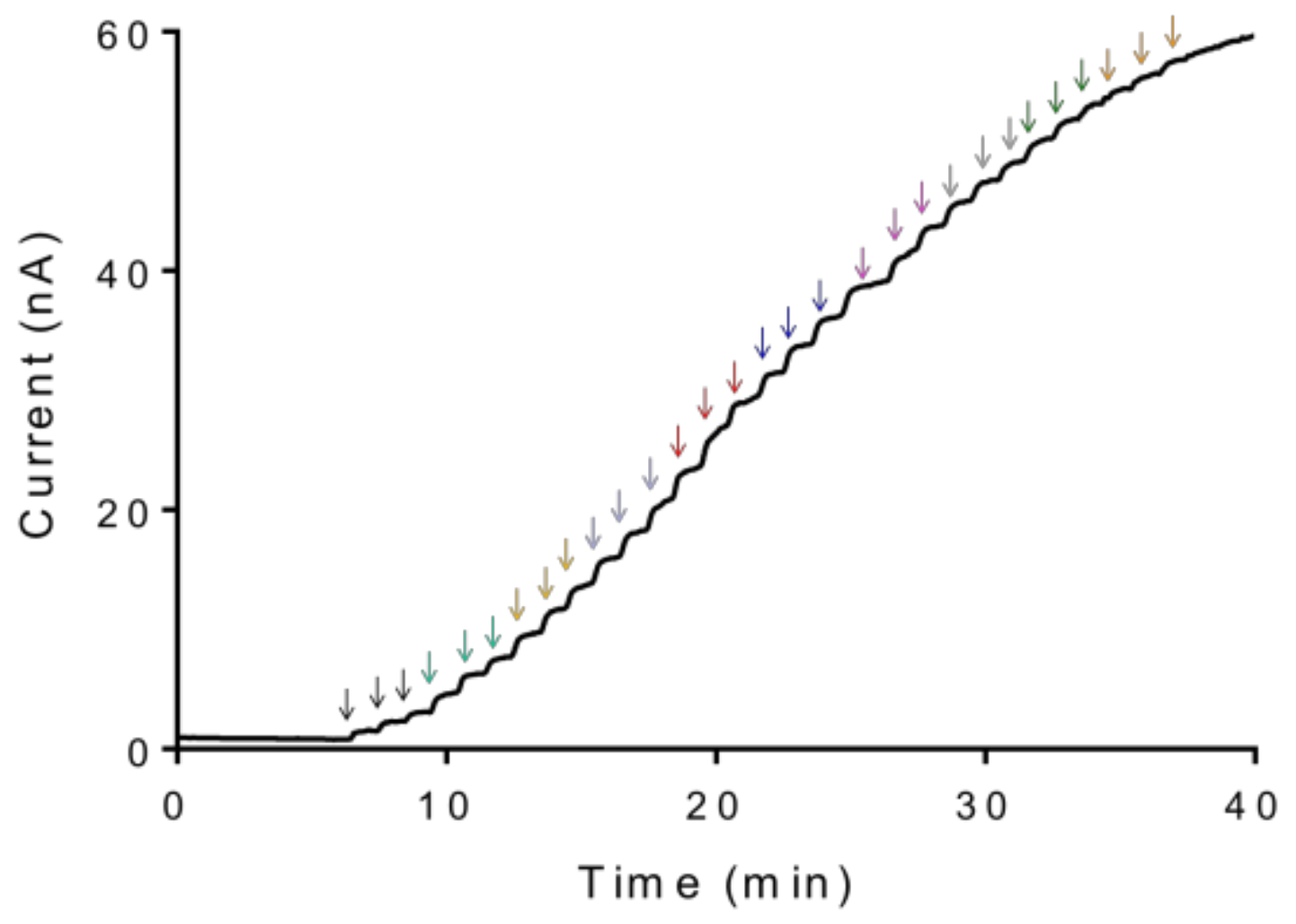

Figure 2. Representative amperogram shows the biosensor response to glutamate at an applied potential of $+0.6 \mathrm{~V}$ vs $\mathrm{Ag} / \mathrm{AgCl}$. Arrows indicated with color coding are to indicate each of the individual concentrations tested in triplicate at concentrations of 5, 10, 15, 20, 25, 30, 35, 40, 45, and $50 \mu \mathrm{M}$, respectively. 
A.

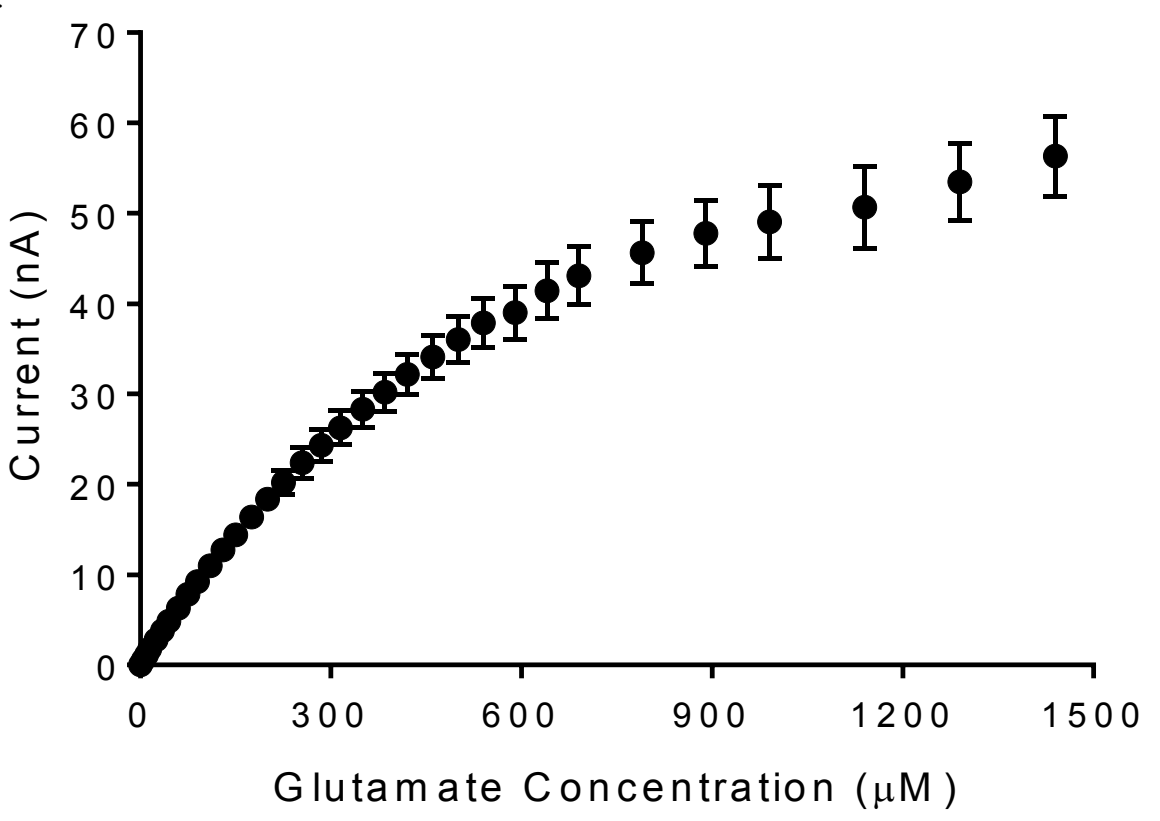

B.

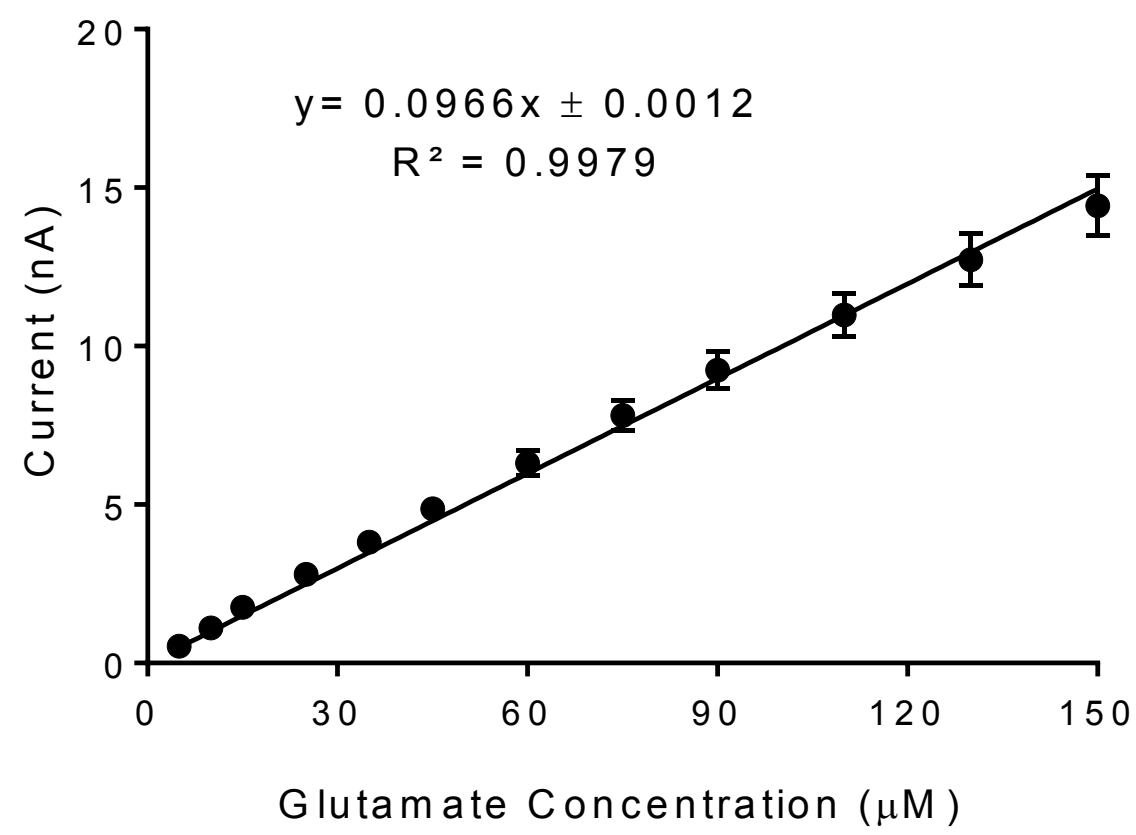

Figure 3. (A) Glutamate biosensor calibration curve showing the sensor performance to glutamate concentrations from $5 \mu \mathrm{M}$ to $1500 \mu \mathrm{M}$. (B) Biosensor has a linear range of sensitivity up to $150 \mu \mathrm{M}$. Within the linear range the biosensor reliably measures glutamate with a sensitivity of $0.0966 \mathrm{nA} / \mu \mathrm{M}$. 


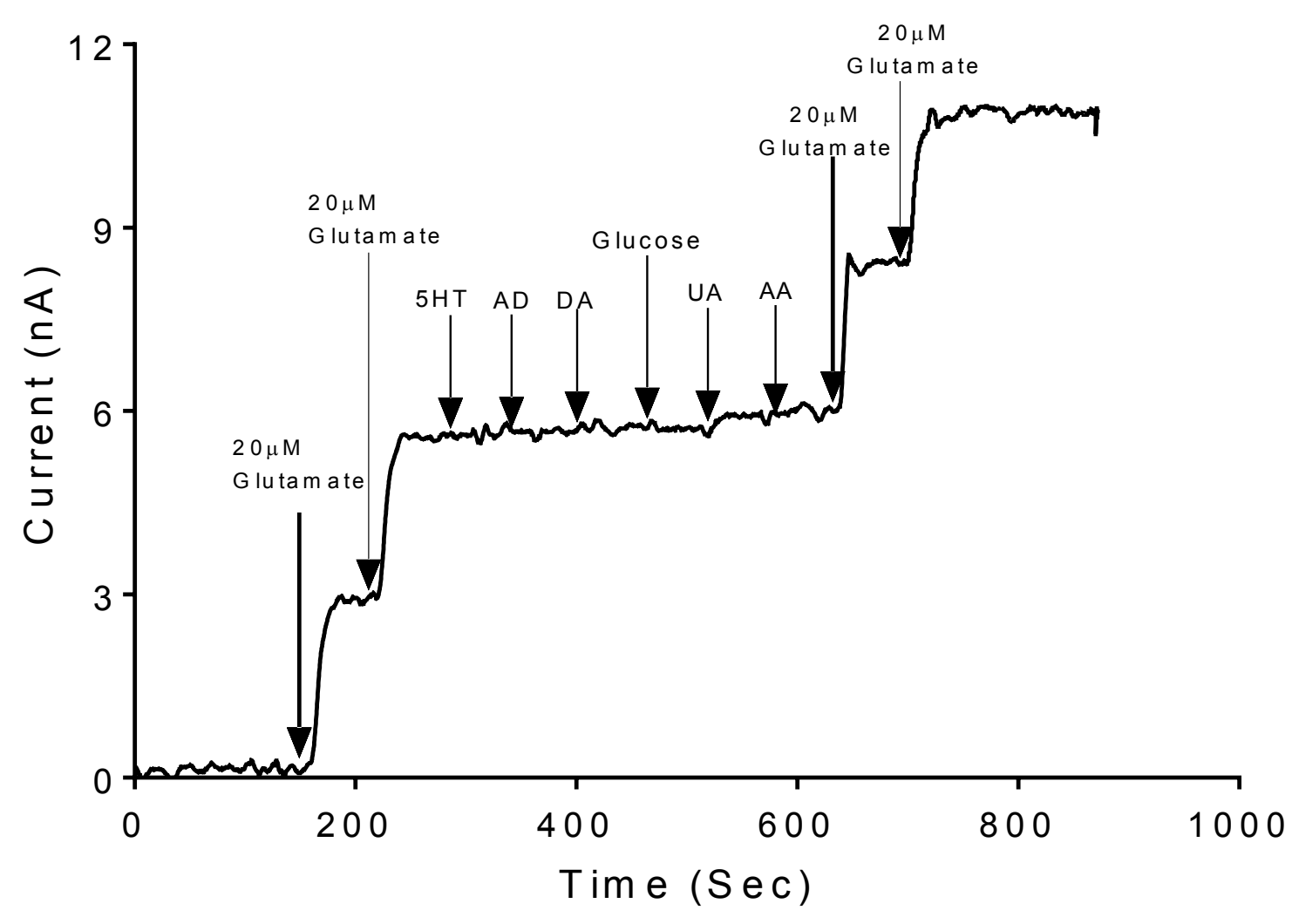

Figure 4. Glutamate biosensor in vitro selectivity testing. Following two injections of $20 \mu \mathrm{M}$ glutamate, a series of species commonly present in the CNS were injected to the electrochemical cell in order to ensure that the biosensor responds only to glutamate. The following species were used: $100 \mathrm{nM}$ serotonin (5HT), $1 \mu \mathrm{M}$ adenosine (AD), $1 \mu \mathrm{M}$ dopamine (DA), $0.5 \mathrm{mM}$ glucose, $100 \mu \mathrm{M}$ uric acid (UA), and $200 \mu \mathrm{M}$ ascorbic acid (AA). Current response to $20 \mu \mathrm{M}$ glutamate prior to exposure to these interferents $(0.135 \mathrm{nA} / \mu \mathrm{M})$ did not change significantly afterwards $(0.123 \mathrm{nA} / \mu \mathrm{M})$. 


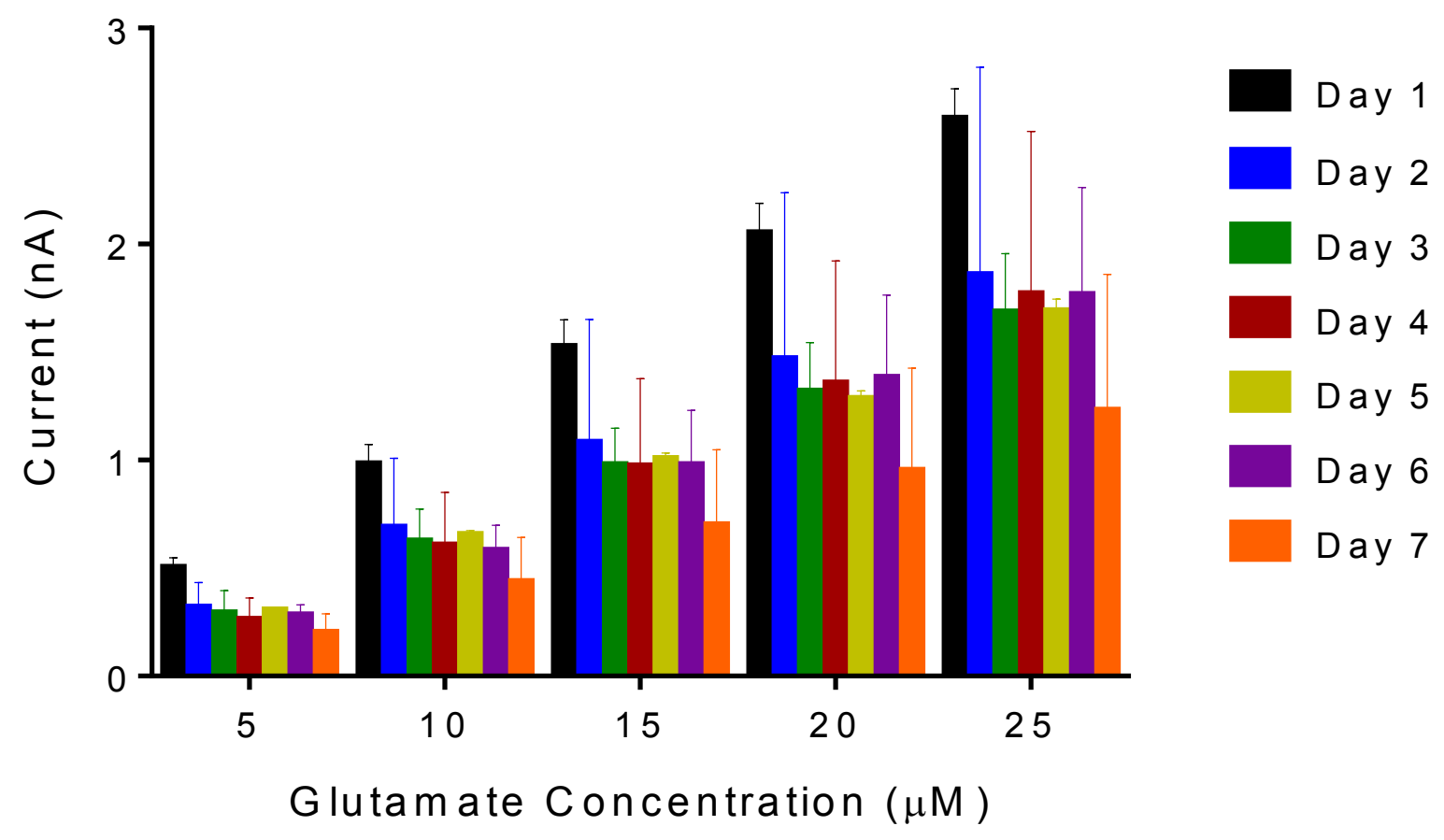

Figure 5. Shelf life stability of glutamate biosensor. Data shows sensitivity of biosensors from Day 1 to Day 7 (Day 0 was the day of enzyme deposition). Average sensitivity (n=3) decreases $55 \%$ from Day 1 to Day 7 when stored under dry conditions. 


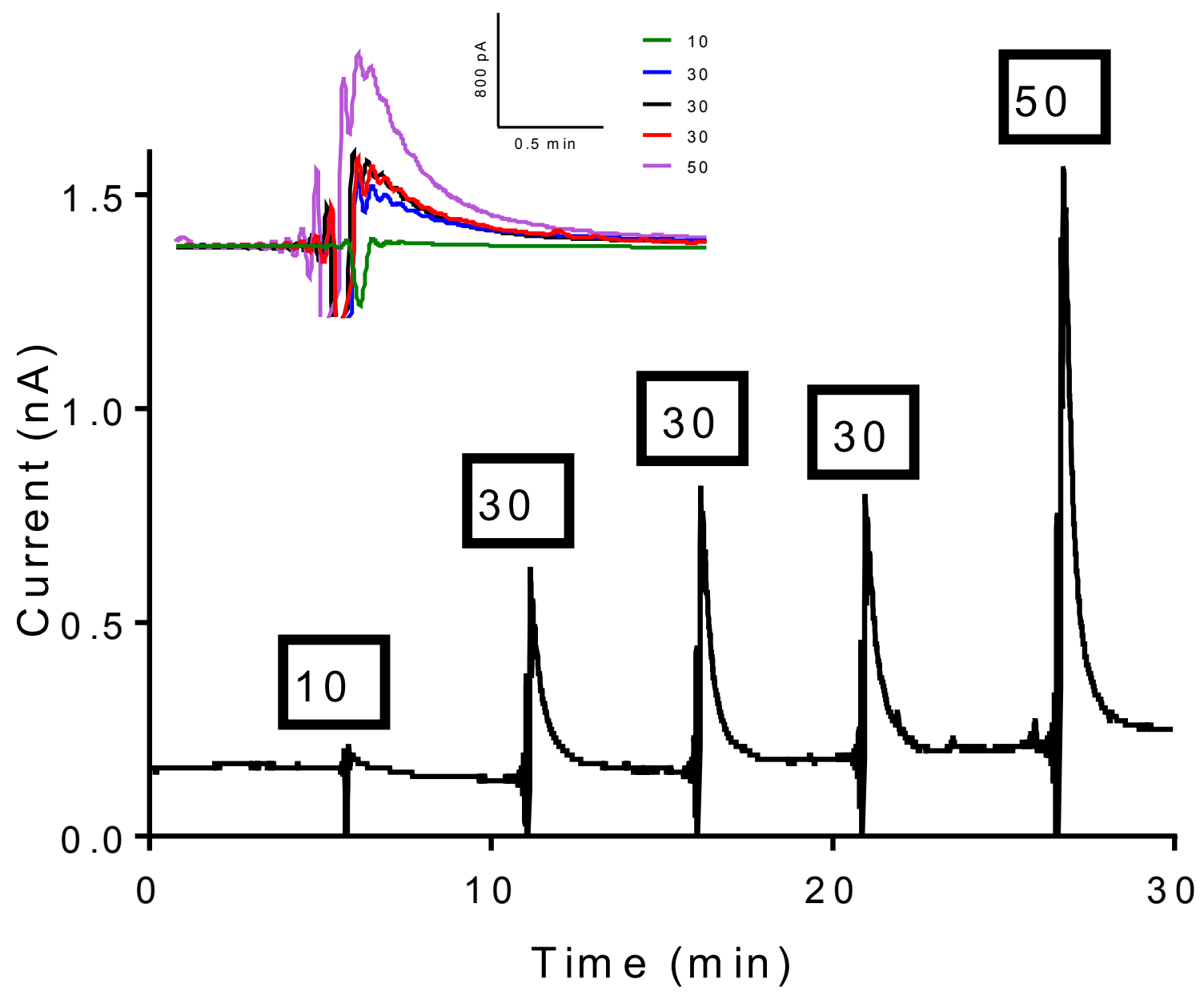

Figure 6. Stimulated glutamate data in rat subthalamic nucleus brain slice. Representative amperogram depicting stimulation of glutamate in the subthalamic nucleus of rat brain slices.

The tissue was stimulated five times with pulse rates of: 10, 30, 30, 30, and 50. Inset overlays the stimulations at a smaller timescale, allowing for comparison of pulse rates. 

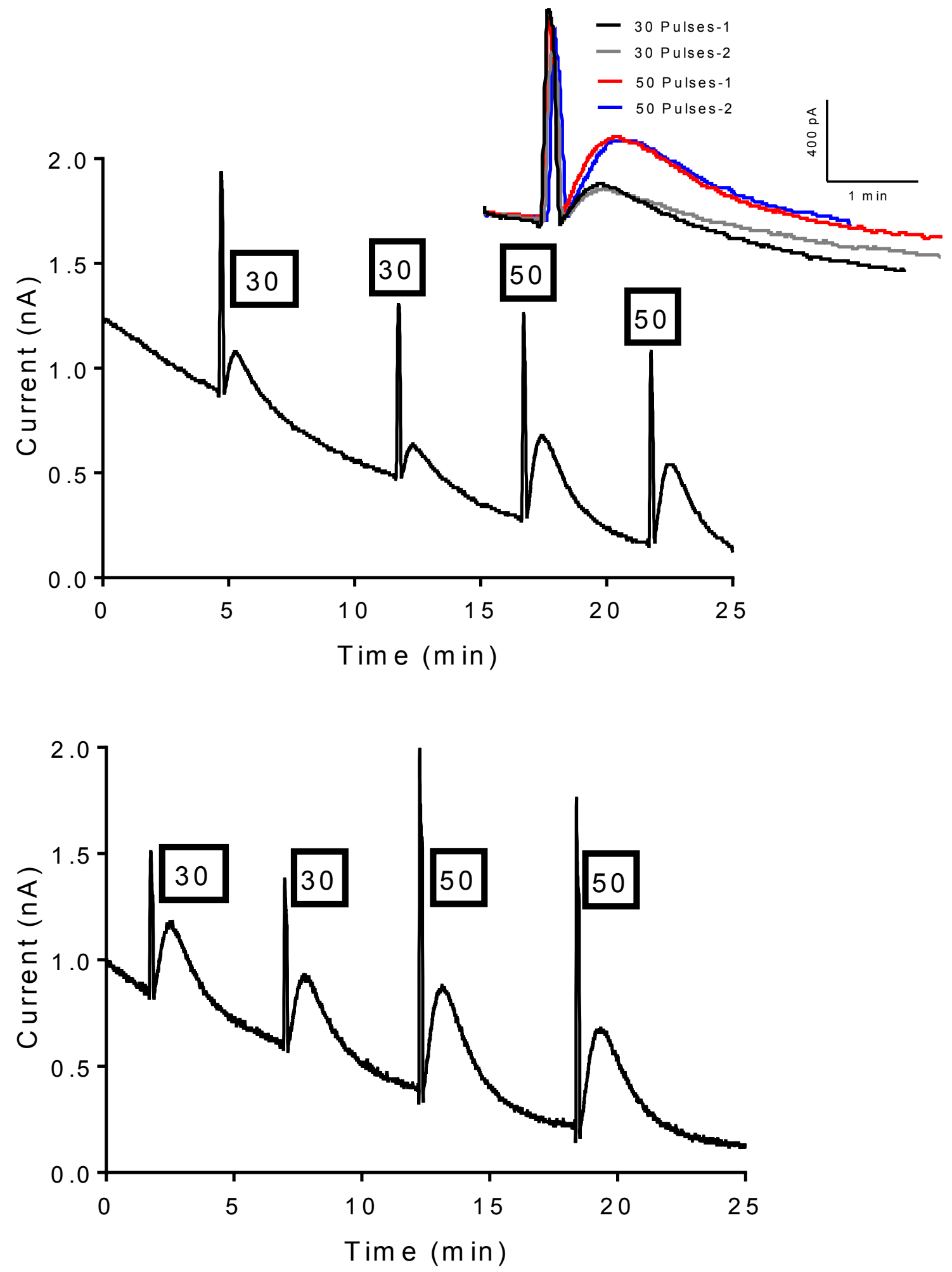

Figure 7. Stimulated glutamate data in rat subthalamic nucleus in vivo. Two representative amperograms depicting stimulation of glutamate in the rat subthalamic nucleus in vivo. In both tissue slices, the tissue was stimulated four times with pulse rates of: $30,30,50$, and 50. Inset overlays the stimulations at a smaller timescale, allowing for comparison of pulse rates. Sharp spike prior to peak glutamate response is electrical noise. 
A.

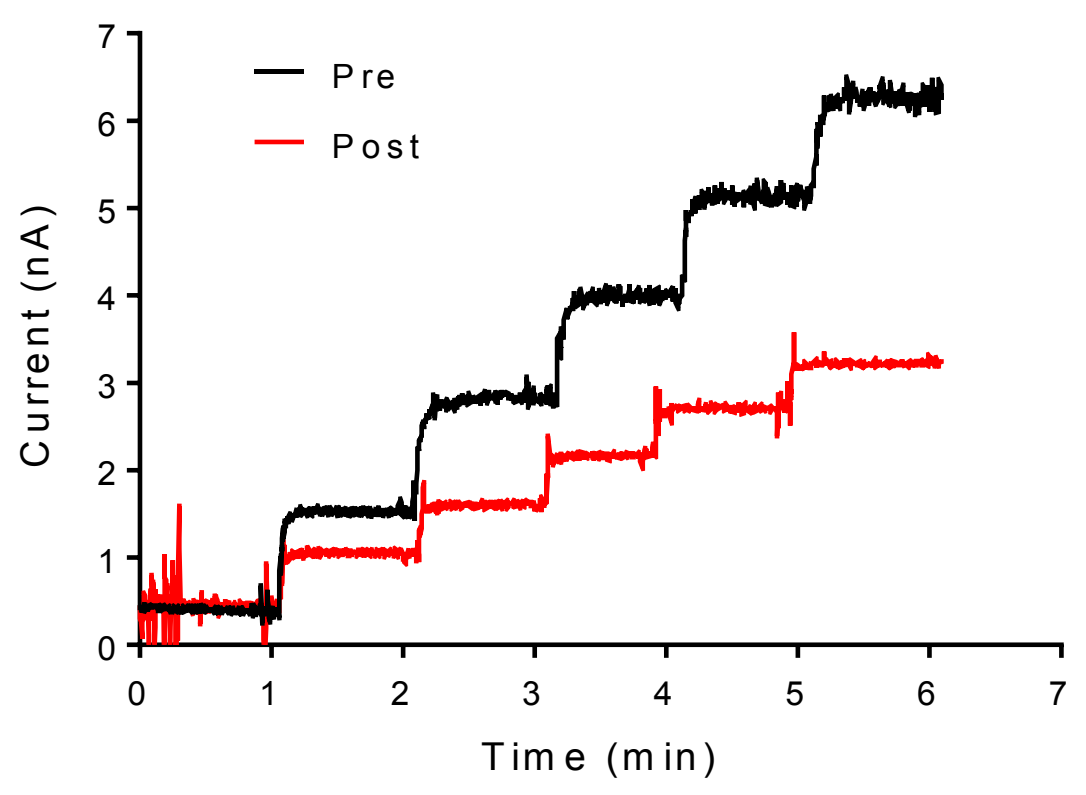

B.

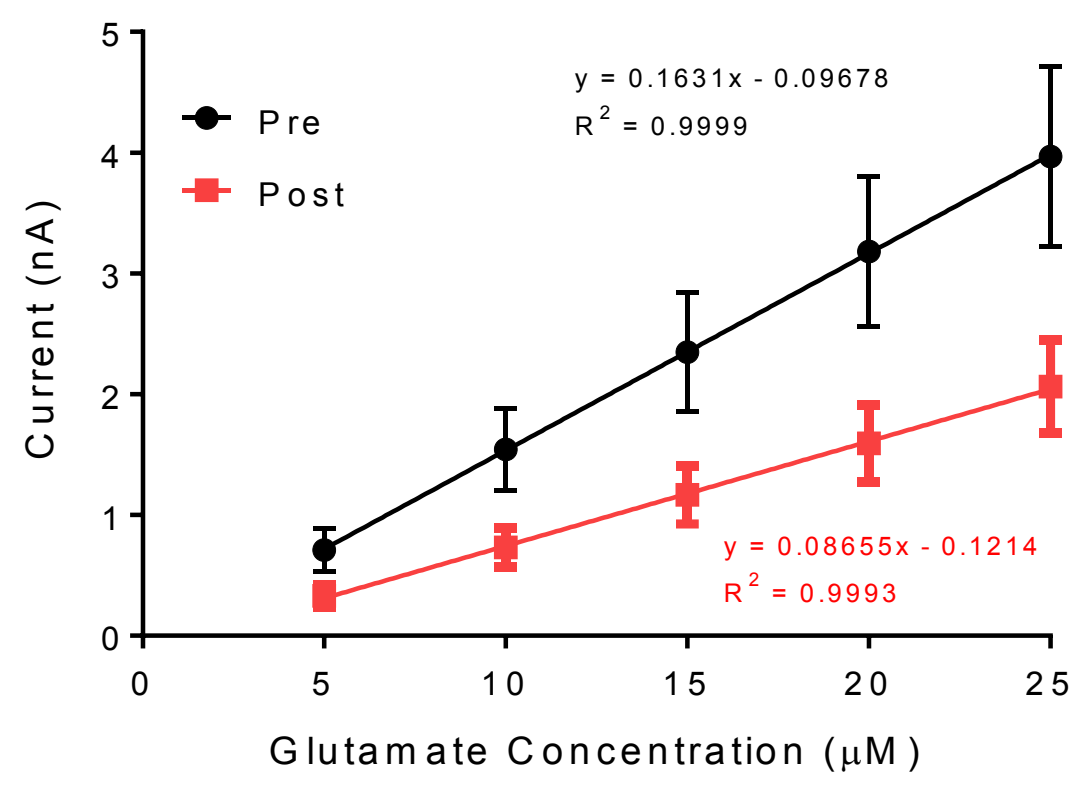

Figure 8. Calibrations of biosensors before and after use in brain tissue shows a reduction in sensitivity. (A) Representative amperograms of calibrations both prior to and after use in brain tissue. (B) Summarized data $(n=4)$ with linear regression of pre-calibrations and post-calibrations both with use in brain slices and in vivo. Biosensor remains responsive to glutamate with $47 \%$ decrease in sensitivity. 